Gynäkologische Endokrinologie 2009 · 7:4 DOI 10.1007/s10304-008-0290-5

Online publiziert: 8. Februar 2009

(c) Springer Medizin Verlag 2009

T. Strowitzki ${ }^{1} \cdot$ M. Ludwig ${ }^{2}$

${ }^{1}$ Abt. für Gynäkologische Endokrinologie und Fertilitätsstörungen, Universitätsfrauenklinik, Heidelberg

${ }^{2}$ Zentrum für Hormon- und Stoffwechselerkrankungen, gynäkologische Endokrinologie und Reproduktionsmedizin, Endokrinologikum Hamburg

\title{
Haut, Haar und Hormone
}

„Blickdiagnose“ auf die richtige Spur führen. In ihrer ganzen Vielfalt stellt PD Dr. Janssen aus Hamburg die internistischen Erkrankungen vor, an die man auch als Frauenarzt denken sollte. Thema für die Gynäkologie! Die Haut und das Haar sind nicht nur Spiegel der Seele, sondern oft genug auch mit ihren Veränderungen erster Hinweis für vielerlei Erkrankungen. Patientinnen stellen sich in nahezu jedem Lebensabschnitt mit Problemen von Haut und Haar in der frauenärztlichen Praxis vor. So klagt fast die Hälfte der Achtzehnjährigen über Akne bzw. unreine Haut, und später - im reproduktiven Alter - sind Hautveränderungen oft erstes Symptom für endokrine Störungen wie das PCO-Syndrom. Unabhängig vom Lebensabschnitt werden Alopezien von den Frauen als besonders belastend empfunden. Nicht zuletzt führen Alterungsprozesse der Haut postmenopausale Frauen oft in der Hoffnung zum Arzt, dem hormonell Einhalt gebieten zu können.

\section{( ) Haut und Haare können erste Hinweise auf Erkrankungen geben}

Wir haben uns deshalb dieses aktuelle Thema vorgenommen, um einmal profund das aktuelle Wissen darzustellen und gute Argumentationshilfen für die tägliche Praxis zu geben.

Professor Hans Wolff aus München hat sicher die größte Erfahrung, was die auch in Studien nachgewiesenermaßen erfolgreiche Therapie mit Minoxidil bei der Alopezie anbelangt. Auch stellt er sehr genau die anderen Präparate dagegen, von denen offensichtlich nur den antiandrogenen Antikonzeptiva eine größere Bedeutung zukommt.

Internistische Erkrankungen spiegeln sich häufig an der Haut. Hier kann die

\section{$\checkmark$ Antiandrogene Substanzen spielen in der Dermatokosmetik eine zunehmend wichtige Rolle}

Hormonelle Antikonzeption dient heute bei Weitem nicht nur dem Empfängnisschutz. Moderne Antikonzeptiva haben sich zu „Lifestyle-Medikamenten“ entwickelt - um einen leider sehr strapazierten Ausdruck zu verwenden. So spielen gerade in der Dermatokosmetik antiandrogene Substanzen in hormonellen Antikonzeptiva eine entscheidende Rolle. Aus dermatologischer Sicht bewertet Frau Professor Kerscher ihre Einsatzmöglichkeiten.

Letztlich wird eine Vielzahl von Präparaten in der sog. ,ästhetischen Endokrinologie" verwendet. Professor Zouboulis aus Dessau befasst sich seit vielen Jahren mit dieser Fragestellung. Die Wirkung vieler dieser Präparate ist nicht bewiesen, aber wir sehen, dass mit einigen durchaus Effekte zu erzielen sind. So sind Wirkungen von Östrogenen auf die Haut sicher, was aber nicht bedeutet, dass sich hierdurch eine Indikationserweiterung der klassischen Hormonersatztherapie bzw. besser Hormontherapie ergibt. Gerade in der ästhetischen Endokrinologie sind die Übergänge zur Anti-Aging-Medizin sicher fließend, und es ist eine zentrale Aufgabe, zwischen „Sinn und Unsinn“ zu unterscheiden, wie wir es seit vielen Jahren fordern. Für diese Diskussion wird der Beitrag von Zouboulis wertvolle Hilfestellung leisten.
Wir hoffen, dass dies ein für Sie alle besonders interessantes Heft ist. Gerade zu diesen Fragen kursieren unter unseren Patientinnen und Patienten Vorstellungen, die wir sicher in den Bereich von Mythen verlagern können. Um ihnen auch sicher zu vermitteln, dass sie mit ihren Problemen in einer ausführlichen Beratung ernst genommen werden, die Sinnvolles von Unsinnigem unterscheidet, dafür bedarf es einer detaillierten Kenntnis des aktuellen Wissensstandes.

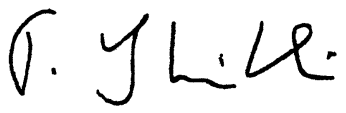

Prof. Dr. Thomas Strowitzki

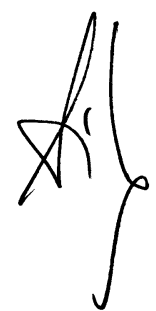

Prof. Dr. M. Ludwig

\section{Korrespondenzadresse}

Prof. Dr. T. Strowitzki

Abt. für Gynäkologische Endokrinologie und Fertilitätsstörungen, Universitätsfrauenklinik Voßstraße 9, 69115 Heidelberg thomas.strowitzki@med.uni-heidelberg.de

Prof. Dr. M. Ludwig

Zentrum für Hormon- und Stoffwechselerkrankungen, gynäkologische Endokrinologie und Reproduktionsmedizin, Endokrinologikum Hamburg Lornsenstraße 6, 22767 Hamburg Michael.Ludwig@endokrinologikum.com 